\title{
COMPLETION FUNCTORS FOR CAUCHY SPACES
}

\author{
R. FRIC and D.C. KENT \\ Vysoká skola dopravná \\ Katedra matematiky F SET \\ Zilina, Ceskoslovensko \\ Department of Mathematics \\ Washington State University \\ Pullman, Washington 99164 U.S.A.
}

(Received April 5, 1979)

ABSTRACT. Completion functors are constructed on various categories of Cauchy Spaces by forming the composition of Wyler's completion functor with suitable modification functors.

KEY WORDS AND PHRASES. Cauchy Space, Cauchy Filter, Completion Functor, Modification Functor.

1980 MATHEMATICS SUBJECT CLASSIFICATION CODES. 54E15, 54A20, $28 A 35$.

1. INTRODUCTION.

Background information on Cauchy spaces and Cauchy space completions is available in references [3], [4], and [8]. However a review of this material will be given in this preliminary section.

A Cauchy space $(X, C)$ is a pair consisting of a set $X$ and a collection of filters $C$ on $X$ which satisfy the following conditions: 
1. For each $x \in \mathrm{X}, \dot{\mathrm{x}} \in \mathcal{C}$, where $\dot{\mathrm{x}}$ denotes the fixed ultrafilter generated by $\{x\}$;

2. If $z \in C$ and $\& \geq \xi$, then $\& \in C$;

3. If $\& \in \mathcal{C}$ and $\vee \&$ exists, then $\cap \& \in C$.

If $(X, C)$ is a Cauchy space, then the set $\mathcal{C}$ is called a Cauchy structure and its elements Cauchy filters. If $(\mathrm{X}, \mathrm{C})$ and $(\mathrm{Y}, \mathbb{A})$ are Cauchy spaces, then $(\mathrm{X}, \mathrm{C})$ is finer than $(\mathrm{Y}, \mathrm{A})(\operatorname{denoted}(\mathrm{Y}, \mathrm{A}) \leq(\mathrm{X}, \mathrm{C}))$ if $\mathrm{X}=\mathrm{Y}$ and $\mathrm{C} \subset d$.

For each Cauchy space $(\mathrm{X}, \mathrm{C})$, there is an associated convergence structure ${ }^{q_{C}}$ on $x$ defined as follows: $\rightarrow x$ in $\left(x, q_{C}\right)$ if $\dot{x} \cap \in C$. A Cauchy space is said to be Hausdorff if each filter converges in $\left(\mathrm{X}, \mathrm{q}_{\mathrm{C}}\right)$ to at most one point. It will be assumed throughout this paper that all Cauchy spaces are Hausdorff unless otherwise indicated.

A Cauchy space $(X, C)$ is complete if each Cauchy filter converges. We shall regard the terms "complete Cauchy space" and "convergence space" as interchangeable; an axiomatization of "convergence space" is given in [8] .

A Cauchy subspace $(Y, D)$ of a Cauchy space $(X, C)$ is a subset $Y$ of $X$ equipped with a Cauchy structure

$$
\theta=\left\{F^{\prime} \text { is a filter on } Y, F^{\prime}\right\} \text {, where }
$$

denotes the filter generated on $\mathrm{X}$ by (considered as a filter base on $\mathrm{X}$ ). If $(X, C)$ is a complete Cauchy space (i.e. convergence space), then it will be necessary to distinguish between a convergence subspace (a subspace in the usual convergence space sense) and a Cauchy subspace (with the meaning defined above). Note that if $(\mathrm{Y}, \mathrm{P})$ is a convergence subspace and $(\mathrm{Y}, \mathbb{Q})$ a Cauchy subspace of a complete Cauchy space $(X, C)$, then $q_{d}=p$.

If $(X, C)$ and $(Y, Q)$ are Cauchy spaces, then a function $f:(X, C) \rightarrow(Y, Q)$ is said to be Cauchy-continuous if $\mathrm{f}(\boldsymbol{F}) \in \mathbb{D}$ whenever $\in \mathbb{C}$. Throughout this paper, the term map will be used exclusively to denote a Cauchy-continuous function. The terms Cauchy-embedding and Cauchy-homeomorphism are defined 
in the obvious way.

For any Cauchy space $(X, C)$, an equivalence relation among Cauchy filters is defined as follows: If $\&, \notin C$, then $\sim \&$ if $\cap \& \in C$. For

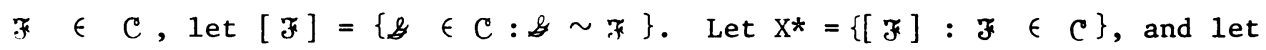
$j$ be the function defined by $: j(x)=[\dot{x}]$, for all $x \in x$. Note that $j$ is injective under our assumption that $(X, C)$ is Hausdorff.

A completion $\left(\left(X^{\prime}, C^{\prime}\right), h\right)$ of a Cauchy space $(X, C)$ consists of a complete Cauchy space $\left(X^{\prime}, C^{\prime}\right)$ and a Cauchy-embedding $h:(X, C) \rightarrow\left(X^{\prime}, C^{\prime}\right)$ such that ${ }^{c l_{q^{\prime}}} h^{h(X)}=X^{\prime} \cdot \quad$ (Notation: ${ }^{c 1} q_{q}$ denotes the closure operation for a convergence structure q.) If the last part of the preceding definition is weakened by stating, instead, that some ordinal iteration of the closure of $h(X)$ equals $X^{\prime}$, then $\left(\left(X^{\prime}, C^{\prime}\right), h\right)$ will be called a weak completion of $(X, C)$. A completion $\left(\left(X^{\prime}, C^{\prime}\right), h\right)$ of $(X, C)$ is said to be strict if the following additional condition is satisfied: If $\epsilon C^{\prime}$, then there is \& $\in C$ such that F $\geq \mathrm{cl}_{\mathrm{q}_{\mathrm{C}}} \mathrm{h}(\&)$. If $\left(\mathrm{X}^{\prime}, \mathrm{C}^{\prime}\right)$ is a topological space, then completion, strict completion, and weak completion are equivalent concepts, but in general they are distinct.

Two completions $\left(\left(X^{\prime}, C^{\prime}\right), h\right)$ and $\left(\left(X^{\prime \prime}, C^{\prime \prime}\right), k\right)$ of $(X, C)$ are said to be

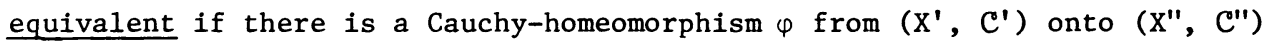
such that the following diagram comutes:

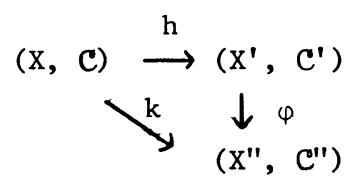

The next result is established in [8].

PROPOSITION 1.1. If $\left(\left(X^{\prime}, C^{\prime}\right), h\right)$ is a completion of a Cauchy space $(\mathrm{X}, \mathrm{C})$, then there is a complete Cauchy structure $\mathrm{C}^{\prime \prime}$ on the set $\mathrm{X}^{*}$ of Cauchy equivalence classes relative to $(X, C)$ such that $\left(\left(X^{\prime}, C^{\prime}\right), h\right)$ and $\left(\left(X^{*}, C^{\prime \prime}\right), j\right)$ 
are equivalent completions.

Let $\mathrm{CHY}$ be the category with Cauchy spaces as objects and maps (i.e., Cauchy-continuous functions) as morphisms. Let $\mathrm{LCH}$ be any full subcategory of $\mathrm{CHY}$, and let $\mathrm{LCH}^{*}$ be the full subcategory of complete objects in $\mathrm{LCH}$.

A completion functor $\mathrm{F}$ on $\mathrm{LCH}$ is a covariant functor $\mathrm{F}: \mathrm{LCH} \rightarrow \mathrm{LCH}$ * which satisfies the following conditions:

1. For each $(\mathrm{X}, \mathrm{C}) \in \mathrm{LCH}$, there is a Cauchy-embedding $i_{\mathrm{F}}:(\mathrm{X}, \mathrm{C}) \rightarrow$ $F(X, C)$ such that $\left(F(X, C), i_{F}\right)$ is a completion of $(X, C)$.

2. If $\mathrm{f}:(\mathrm{X}, \mathrm{C}) \rightarrow(\mathrm{Y}, \mathbb{\theta})$ is a map, with $(\mathrm{X}, \mathrm{C}) \in \mathrm{LCH}$ and $(\mathrm{Y}, \theta) \in \mathrm{L} \mathrm{CH}^{*}$, then there is a unique map $\bar{f}: F(X, C) \rightarrow(Y, A)$ such that the following diagram commutes:

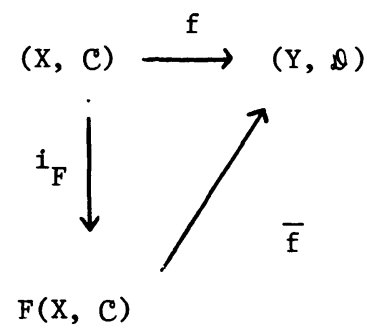

If $\mathrm{F}$ is a completion functor on $\mathrm{LCH}$, then it follows that any map $f:\left(x_{1}, C_{1}\right) \rightarrow\left(x_{2}, C_{2}\right)$ between objects in $L C H$ has a unique Cauchy-continuous extension $\overline{\mathrm{f}}:\left(\mathrm{X}_{1}, \mathrm{C}_{1}\right) \rightarrow \mathrm{F}\left(\mathrm{X}_{2}, \mathrm{C}_{2}\right)$, and $\overline{\mathrm{f}}=\mathrm{F}(\mathrm{f})$. Thus, two completion functors $F_{1}$ and $F_{2}$ on the same category $L C H$ are equivalent in the sense that, for each $(X, C) \in \underbrace{L C H}$, the completions $\left(F_{1}(X, C), i_{F_{1}}\right)$ and $\left(F_{2}(X, C), i_{F_{2}}\right)$ are equivalent.

A full subcategory $\mathrm{LCH}$ of $\mathrm{CHY}$ which admits a completion functor will be called a completion subcategory of C HY. Examples of completion subcategories are the categories of $\mathrm{C}^{\wedge}$-embedded spaces and sequentially regular spaces described in [3]. These and other examples emerge as special cases in the general theory developed in this paper. 


\section{WYLER'S COMPLETION FUNCTOR.}

Ellen Reed, [8], constructed a family of completions for any Cauchy space ( $X, C)$. One member of this family, called Wyler's completion, is the Cauchy space formulation of a completion developed by 0 . Wyler for uniform convergence spaces in [10]. Wyler's completion defines a completion functor whose domain is the whole category CHY (i.e., CHY is a completion subcategory of itself); this completion functor forms the foundation for the completion theory developed in this paper. Wyler's completion (but not so named) also appears in a recent abstract by Redfield [7].

Given $(X, C) \in \underbrace{C H Y}$, we define a convergence structure $q^{*}$ on the set $X^{*}$ of Cauchy equivalence classes as follows: A filter $\sharp$ on $X^{*} q^{*}$ - converges to $\alpha$ in $X^{*}$ if there is a filter $\in \alpha$ such that $\sharp \geq(j(j)) \cap \dot{\alpha}$. Let $C *$ be the complete Cauchy structure on $X^{*}$ consisting of all $q^{*}$ - convergent filters. Then it is easy to verify that $\left(\left(X^{*}, C^{*}\right), j\right)$ is a strict completion of $(X, C)$, and that the only member of $C^{*}$ containing $X^{*}-j(X)$ are fixed ultrafilters.

PROPOSITION 2.1. If $\mathrm{f}:(\mathrm{X}, \mathrm{C}) \rightarrow\left(\mathrm{X}^{\prime}, \mathrm{C}^{\prime}\right)$ is a map and $\left(\mathrm{X}^{\prime}, \mathrm{C}^{\prime}\right)$ is complete, then there is a unique map $\bar{f}:\left(X^{*}, C^{*}\right) \rightarrow\left(X^{\prime}, C^{\prime}\right)$ such that the following diagram commutes:

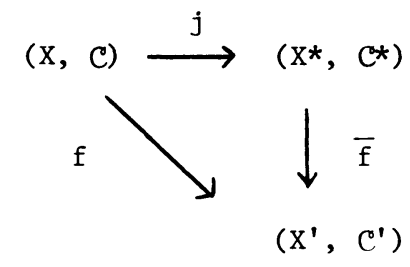

PROOF. If $x \in X$, define $\bar{f}([x])=f(x)$; if $\alpha \in X^{*}-j(X)$, define $f(\alpha)=y$ if there is $\epsilon$ such that $f(x) \rightarrow y$ in $\left(X^{\prime}, C^{\prime}\right)$. It is a routine matter to verify that $\bar{f}$ is a unique map, and that the above diagram commutes. 
Define the functor $\mathrm{W}: \mathrm{CHY}^{\mathrm{CH}} \rightarrow \mathrm{HY}^{*}$ as follows: If $(\mathrm{X}, \mathrm{C})$ is an object in $\mathrm{CHY}^{\mathrm{H}}$ then $\mathrm{W}(\mathrm{X}, \mathrm{C})=\left(\mathrm{X}^{*}, \mathrm{C}^{*}\right)$; if $\mathrm{f}:(\mathrm{X}, \mathrm{C}) \rightarrow\left(\mathrm{X}^{\prime}, \mathrm{C}^{\prime}\right)$ is a morphism in CHY, then $W(f)=\bar{f}$, where $\bar{f}: W(X, C) \rightarrow W\left(X^{\prime}, C^{\prime}\right)$ is the unique extension map whose existence is guaranteed by Proposition 2.1. It is clear from Proposition 2.1 that $\mathrm{W}$ is a completion functor on $\mathrm{CHY}$; W will be called Wyler's completion functor.

PROPOSITION 2.2. Let $(\mathrm{Y}, \theta)$ be a subspace of a Cauchy space $(\mathrm{X}, \mathrm{C})$, and let id $: \mathrm{Y} \rightarrow \mathrm{X}$ be the identity embedding. Then the extension $\overline{\mathrm{id}}: \mathrm{W}(\mathrm{Y}, \mathbb{\theta}) \rightarrow$ $W(X, C)$ is injective.

PROOF. The theorem is an immediate consequence of the following observation. If $\bar{z}$ and $\&$ are filters on $\mathrm{Y}$ belonging to $\mathcal{A}$, and if ${ }^{\prime}$ and $\&$ are the filters on $X$ generated by and respectively, then $F^{\prime} \cap \in C$ if and only if $\cap \& \in C$.

Wyler's completion does not, in general, preserve such important properties as uniformizability, regularity, or total boundedness. By constructing completion functors on certain subcategories of $\mathrm{CHY}$, one obtains completions which preserve all of the defining properties of the subcategories, and sometimes other properties as well. A general approach to obtaining completion subcategories of $\mathrm{CHY}$ and their completion functors by means of modification functors is described in the next section.

As a matter of convenience and notational simplicity and since it entails no loss of generality, we shall adopt the following convention for the remainder of this paper: For each $(X, C) \in C H Y$ and $x \in X$, we shall identify $x$ with the element $[\dot{x}]$ in $X^{*}$, and consider $(X, C)$ to be a subspace of $W(X, C)$.

\section{MODIFICATION FUNCTORS.}

Our goal is to describe completion subcategories of $\mathrm{CH}$ which are maximal relative to some Cauchy space property. This is accomplished for properties 
which can be characterized by means of modification functors subject to certain restrictions. Each such modification functor $M$ gives rise to a completion subcategory $\underbrace{\mathrm{MCHY}}$ of $\mathrm{CHY}$, and the composite functor $\mathrm{MW}$ is the unique completion $\mathrm{MW}$ is the unique completion functor on MCHY .

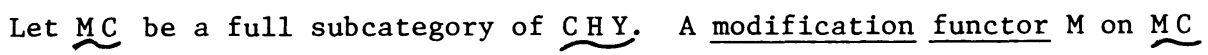
is a convariant functor $M: \underline{M C} \rightarrow \underline{\mathrm{CHY}}$ with the following properties:

1. For each object $(X, C) \in M C, M(X, C)$ and $(X, C)$ have the same underlying set;

2. For each object $(X, C) \in \underbrace{M C}, M(M(X, C))=M(X, C)$;

3. For each morphism $\mathrm{f} \in \mathrm{MC}, \mathrm{M}(\mathrm{f})=\mathrm{f}$.

If $M$ is a modification functor on $\underline{M C}$, then $(X, C) \in \underline{M C}$ is called an M-space if $M(X, C)=(X, C)$. In what follows, we shall be interested in modification functors which are subject to the following additional conditions.

(L) For each object $(X, C) \in M C, M(X, C) \leq(X, C)$.

(H) If $(X, C) \in \underline{M C}$ and $(Y, \infty)$ is a subspace of $(X, C)$, then $(\mathrm{Y}, \theta) \quad \mathrm{MC}$. If, in addition, $(\mathrm{X}, \mathrm{C})$ is an $\mathrm{M}$-space, then $(\mathrm{Y}, \theta)$ is also an M-space.

(C) If $(X, C) \in \underline{M C}$, then $W(X, C) \in \underline{M C}$ and $M W(X, C)$ is complete.

For the remainder of this section, we assume that $M: \underline{M C} \rightarrow \underline{C H Y}$ is a modification functor which satisfies conditions (L), (H), and (C).

PROPOSITION 3.1. a. If $(\mathrm{X}, \mathrm{C})$ and $(\mathrm{X}, \mathbb{Q})$ are in $\mathrm{MC}$ and $(\mathrm{X}, \mathrm{C}) \leq(\mathrm{X}, \mathbb{Q})$ then $M(X, C) \leq M(X, \theta)$

b. If $(X, C) \in M C$, then $M(X, C)$ is the finest $M$-space coarser than $(\mathrm{X}, \mathrm{C})$

PROOF. a. Follows immediately by applying $M$ to the identity map from $(X, C)$ to $(X, \infty)$.

b. Follows easily from (a). 
For any object $(X, C) \in \underline{M C}$, we define $M^{\wedge}(X, C)$ to be the Cauchy subspace of MW(X, C) whose underlying set is X. It follows from (L), (H), and (C) that $M(X, C)$ is an $M$-space, and therefore $M^{\wedge}(X, C) \leq M(X, C)$ follows by Proposition 3.1 (a). Let $\underline{M C H Y}$ denote the full subcategory of $\underline{M C}$ whose objects are those Cauchy spaces $(X, C)$ such that $(X, C)=M^{\wedge}(X, C)$.

THEOREM 3.2. The following statements about a Cauchy space $(\mathrm{X}, \mathrm{C})$ are equivalent.

1. $(\mathrm{X}, \mathrm{C}) \in \underline{\mathrm{MCHY}}$.

2. (X, C) is a subspace of a complete M-space.

3. (X, C) has a weak M-space completion.

PROOF. The only non-obvious implication is $(2) \Rightarrow(1)$. Assume that $(\mathrm{X}, \mathrm{C})$ is a subspace of a complete $\mathrm{M}$-space $(\mathrm{Y}, \mathbb{\theta})$. Then the identity map id $:(X, C) \rightarrow(Y, \theta)$ has an injective extension map $\overline{\text { id }}: \mathrm{W}(\mathrm{X}, \mathrm{C}) \rightarrow \mathrm{W}(\mathrm{Y}, \theta)=$ $(\mathrm{Y}, \mathbb{A})$ by Proposition 2.2. By Proposition 3.1 (a), $\overline{\mathrm{Id}}: \mathrm{MW}(\mathrm{X}, \mathrm{C}) \rightarrow(\mathrm{Y}, \mathbb{A})$ is also an injective map. Restricting $\mathrm{MW}(\mathrm{X}, \mathrm{C})$ and $(\mathrm{Y}, \mathrm{\theta})$ to $\mathrm{X}$, we obtain $M^{\wedge}(X, C) \geq(X, C)$. But $M^{\wedge}(X, C) \leq(X, C)$ is always true, and therefore $(\mathrm{X}, \mathrm{C}) \in \mathrm{MCHY}$.

THEOREM 3.3. The composite functor $\mathrm{NW}$ is a completion functor on M C HY .

PROOF. Let $(\mathrm{X}, \mathrm{C}) \in \underline{\mathrm{MCHY}}$. In the definition of completion functor, identify $i_{\text {MW }}$ with the identity embedding $j:(X, C) \rightarrow W(X, C)$. Since $(X, C)$ is a dense subspace of $W(X, C),(X, C)=M^{\wedge}(X, C)$ is a subspace of $M W(X, C)$, and $M W(X, C) \leq W(X, C)$, it follows that $(X, C)$ is a dense subspace of MW (X, C). Furthermore, MW (X, C) is complete by condition (C).

Let $f:(X, C) \rightarrow(Y, \&)$ be a map, where $(X, C) \in \underline{M C H Y}$ and $(Y, \theta) \in \underline{M C H Y}$. In the diagram that follows, each unlabeled arrow is the identity map. 


$$
\begin{aligned}
& (\mathrm{X}, \mathrm{C}) \rightarrow \mathrm{W}(\mathrm{X}, \mathrm{C}) \rightarrow \mathrm{MW}(\mathrm{X}, \mathrm{C}) \\
& \mathrm{f} \downarrow \quad \overline{\mathrm{f}} \downarrow \quad \overline{\mathrm{f}} \downarrow \\
& (\mathrm{Y}, \theta) \rightarrow(\mathrm{Y}, \theta) \rightarrow(\mathrm{Y}, \theta)=\mathrm{M}(\mathrm{Y}, \theta)
\end{aligned}
$$

It follows from the universal property of $W$ and our assumptions governing $M$ that each of the above maps is Cauchy-continuous and uniquely determined, and that the diagram commutes. This completes the proof of the theorem.

COROLLARY 3.4. MCHY is the largest category whose objects consist only of M-spaces which forms a completion subcategory of $\mathrm{CHY}$.

PROOF. MCHY is a completion subcategory of CHY by Theorem 3.3. The remainder of the assertion is an immediate consequence of Theorem 3.3.

A Cauchy space $(X, C)$ is $\underline{\text { regular }}$ if $\in \mathcal{C}$ implies $\mathrm{cl}_{\mathrm{q}_{\mathrm{C}}} \boldsymbol{F} \in \mathrm{C}$.

THEOREM 3.5. Let $M$ be a modification functor such that $M(X, C)$ is regular for each $(X, C) \in M C$. If $\left(\left(X^{\prime}, C^{\prime}\right), h\right)$ is a strict completion of an object $(X, C) \in M C H Y$ such that $\left(X^{\prime}, C^{\prime}\right)$ is an M-space, then $\left(\left(X^{\prime}, C^{\prime}\right), h\right)$ is equivalent to the completion $M W(X, \mathcal{C})$.

PROOF. In view of Proposition 1.1, we can assume that $X^{\prime}=X^{*}$ is the set of all Cauchy equivalence classes relative to $(\mathrm{X}, \mathrm{C})$, and, in accordance with our convention that $\mathrm{X}$ is a subset of $\mathrm{X}^{\star}$, we can consider $\mathrm{h}$ to be the identity embedding of $\mathrm{X}$ into $\mathrm{X}^{\prime}$.

From the universal property of the functor $M W$, it follows immediately that $\left(X^{\prime}, C^{\prime}\right) \leq M W(X, C)$. Let $\rightarrow y$ in $\left(X^{\prime}, C^{\prime}\right)$; then by the assumption of strictness there is a filter $\& \rightarrow y$ in $\left(X^{\prime}, C^{\prime}\right)$ such that $x \in \&$ and ${ }^{\mathrm{c}} \mathrm{q}_{\mathrm{C}^{\prime}} \& \leq$. But it is a simple matter to verify that $\mathrm{cl}_{\mathrm{q}_{\mathrm{C}^{\prime}}} \&=\mathrm{cl}_{\mathrm{p}} \&$, where $\mathrm{p}$ is the convergence structure on $X^{*}$ associated with $M W(X, C)$. Since $\mathrm{X} \in \&, \& \rightarrow \mathrm{y}$ in $\mathrm{MW}(\mathrm{X}, \mathrm{C})$, and the regularity of $\mathrm{MW}(\mathrm{X}, \mathrm{C})$ implies that $\mathrm{Cl}_{\mathrm{p}} \& \mathrm{y}$ in $\mathrm{MW}(\mathrm{X}, \mathrm{C})$. Consequently, $\rightarrow \mathrm{y}$ in $\mathrm{MW}(\mathrm{X}, \mathrm{C})$, and the two completions are equivalent. 
We note earlier that topological Cauchy space completions are always strict; this fact yields the following corollary.

COROLLARY 3.6. If $M$ is a modification functor as described in Theorem 3.5, then any topological M-space completion of $(\mathrm{X}, \mathrm{C})$ is equivalent to $\mathrm{MW}(\mathrm{X}, \mathrm{C})$.

We conclude this section by remarking that if $M$ is a modification functor on ${ }^{M C}$ satisfying (H), (L), and (C), then $M^{\wedge}: \underbrace{M C} \rightarrow M C H Y$ is also a modification functor which satisfies $(H),(L)$, and $(C)$. If $(X, C) \in M C$, then $\mathrm{M}^{\wedge}(\mathrm{X}, \mathrm{C})$ can be interpreted, in view of Proposition 3.1 (b), as the finest member of $M C H Y$ coarser than $(X, C)$. In general, the modification functors $M$ and $M^{\wedge}$ are distinct on their common domain category $\stackrel{M}{C}$; this fact is illustrated in Section 4 in the case where $M=R$ is the regular modification functor. However if $(X, C) \in M C H Y$, then $M(X, C)=M^{\wedge}(X, C)$ and $M W(X, C)=$ $M W(X, C)$; thus these two modification functors define the same completion functor.

\section{THE REGULAR COMPLETION FUNCTOR.}

The concepts discussed in the preceding section are illustrated in this section using the regular modification functor $R$ in place of the general modification functor $M$.

If $(X, C) \in C H Y$, let $C_{R}$ be the finest regular Cauchy structure on $X$ which is coarser than $C$; $\mathbb{C}_{R}$ is commonly called the "regular modification" of $C$, although it should be noted that $\left(x, C_{R}\right)$ will not be Hausdorff unless additional restrictions are placed on $(\mathrm{X}, \mathrm{C})$. Let $\mathrm{RC}$ be the full subcategory of ${ }^{\mathrm{CHY}}$ whose objects are Cauchy spaces $(\mathrm{X}, \mathrm{C})$ such that $\left(\mathrm{X}^{*}, \mathrm{C}^{*}{ }_{\mathrm{R}}\right) \in \mathrm{CHY}^{\mathrm{CH}}$,

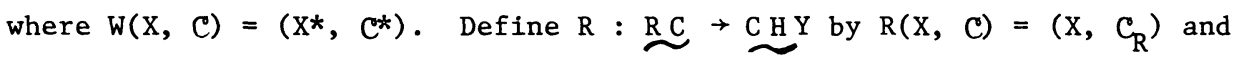
$R(f)=f$ for each object $(X, C)$ and morphism $f$ in $R$. The $\underline{R-s p a c e s}$ are the regular objects in $\mathrm{RC}$. 
PROPOSITION 4.1. $\mathrm{R}$ is a modification functor on $\mathrm{RC}$ which satisfies conditions $(\mathrm{L}),(\mathrm{H})$, and $(\mathrm{C})$.

PROOF. One can verify straightforwardly that $R$ is a modification functor; it is obvious that (L) is satisfied.

Let $(X, C) \in R C$ and let $(Y, Q)$ be a Cauchy subspace of $(X, C)$. Since regularity is known to be herditary for Cauchy spaces, a subspace of an $\mathrm{R}$-space is an $\mathrm{R}$-space. It remains to prove $(\mathrm{Y}, \mathbb{Q}) \in \mathrm{RC}$. Put $\left(\mathrm{Y}^{*}, \mathbb{Q}^{*}\right)=$ $\mathrm{W}(\mathrm{Y}, \mathbb{Q})$ and $\left(\mathrm{X}^{*}, \mathrm{C}^{*}\right)=\mathrm{W}(\mathrm{X}, \mathrm{C})$. Since by Proposition 2.2, the mapping $\overline{\mathrm{id}}: \mathrm{W}(\mathrm{Y}, \mathbb{Q}) \rightarrow \mathrm{W}(\mathrm{X}, \mathrm{C})$ is injective, we can consider $\mathrm{Y}^{*}$ as a subset of $\mathrm{X}^{*}$. Denote by $\mathbb{A}^{\prime}$ (respectively, $\mathbb{Q}^{\prime \prime}$ ) the Cauchy structure for Y inherited from $\left(X^{*}, C^{*}\right)\left(\right.$ respectively, $\left.\left(X^{*}, C^{*}{ }_{R}\right)\right)$. Clearly, $\left(Y^{*}, \theta^{*}\right) \geq\left(Y^{*}, \theta^{\prime}\right) \geq\left(Y^{*}, \theta^{\prime \prime}\right)$, and $\left(Y *, \theta_{R}^{*}\right) \geq\left(Y *, \theta_{R}^{\prime}\right) \geq\left(Y *, \theta_{R}^{\prime}\right)=\left(Y^{*}, \theta^{\prime \prime}\right)$. But the last space is Hausdorff, and hence all finer spaces are Hausdorff, too. Thus $R W(Y, \mathbb{\theta})=$ $\left(Y^{*}, Q{ }_{R}^{*}\right) \in R C$, and so $(H)$ is satisfied.

If $(\mathrm{X}, \mathrm{C}) \in \mathrm{RC}$, then $\mathrm{RW}(\mathrm{X}, \mathrm{C})=\left(\mathrm{X}^{*}, \mathrm{C}^{*}{ }_{\mathrm{R}}\right) \in \mathrm{CH}^{\mathrm{C}}$, and the convergence structure $\mathrm{p}$ on $\mathrm{X}^{*}$ determined by $\mathrm{C}^{*}{ }_{\mathrm{R}}$ is a regular convergence structure. The conplete Cauchy structure $C^{\prime}$ on $X^{*}$ consisting of the p-convergent filters is also a regular Cauchy structure, and $\mathrm{RW}(\mathrm{X}, \mathrm{C}) \leq\left(\mathrm{X}^{*}, \mathrm{C}^{\prime}\right) \leq \mathrm{W}(\mathrm{X}, \mathrm{C})$. Thus $\mathrm{RW}(\mathrm{X}, \mathrm{C})=\left(\mathrm{X}^{*}, \mathrm{C}^{\prime}\right)$ is complete.

By virute of Theorem 3.2, we can characterize the regular completion subcategory RCHY of $\mathrm{CHY}$ as consisting of those Cauchy spaces (X, C) which are Cauchy subspaces of regular convergence spaces. The completion functor $\mathrm{RW}$ on RCHY will be called the regular completion functor. We shall conclude this section with examples which show that there are regular Cauchy spaces in CHY which are not R-spaces, and that there are R-spaces in $\mathrm{RC}$ which are not members of RCHY.

EXAMPLE 4.2. Let $(X, p)$ be a minimal regular topological space which is not compact; an example of such a space is given in [1]. It is also shown in 
[1] that $(X, P)$ cannot be completely regular.

From the results of Section 1 of [5], it follows that there is a regular Cauchy structure $C$ on $X$ compatible with $\mathrm{p}$ such that every ultrafilter on $\mathrm{X}$ is a member of $\mathcal{C}$ (i.e., $C$ is totally bounded), and the non-convergent Cauchy filters form a single equivalence class. Thus $W(X, C)$ is a convergence space one-point compactification of $(\mathrm{X}, \mathrm{C})$.

Suppose RW (X, C) is Hausdorff. Then RW (X, C) would be a compact, regular, Hausdorff convergence space, which is shown in [9] to have the same ultrafilter convergence as a compact, Hausdorff topological space. Let $(X, q)$ be the convergence subspace of $R W(X, C)$ determined by the set $X$. Since $(X, q) \leq(X, p)$, either (X, p) is completely regular, or else there is a completely regular, Hausdorff topological space coarser than $(X, p)$. In either case, the original assumptions about $(X, p)$ are contradicted. Consequently, RW (X, C) cannot be Hausdorff.

We have shown that $(\mathrm{X}, \mathrm{C})$ is a regular member of $\mathrm{CHY}$ which is not in $\mathrm{RC}$, and consequently is not an R-space. More generally, we can assert that $\mathrm{RC}$, is a proper subcategory of the full subcategory of $\mathrm{CHY}$ consisting of those Cauchy spaces whose R-moditications are Hausdorff.

EXAMPLE 4.3. Let $\mathrm{X}$ be an infinite set and a free ultrafilter of $\mathrm{X}$. Let $\mathrm{C}$ consist of all fixed ultrafilters, along with all finite intersections of free ultrafilters, excluding 3 . Thus all ultrafilters are Cauchy except , the associated convergence space $\left(\mathrm{X}, \mathrm{q}_{\mathrm{C}}\right)$ is discrete, $(\mathrm{X}, \mathrm{C})$ is a regular member of $\mathrm{CHY}$, and, as in the preceding example, the non-convergent Cauchy filters form a single equivlence class.

Wyler's completion $\mathrm{W}(\mathrm{X}, \mathrm{C})$ is obtained by adding a single point, call it $\alpha$, to $X$. Considering as a filter on $X \cup\{\alpha\}$, we observe that does not converge to $\alpha$ in $\mathrm{W}(\mathrm{X}, \mathrm{C})$, but does converge to $\alpha$ in $\mathrm{RW}(\mathrm{X}, \mathrm{C})$. Indeed, $\mathrm{RW}(\mathrm{X}, \mathrm{C})$ has the same ultrafilter convergence as the topological one-point 
compactification of the discrete topological space $\left(x, q_{C}\right)$, which implies that (X, C) is an R-space. Since (X, C) is not a Cauchy subspace of $\mathrm{R} W(\mathrm{X}, \mathrm{C})$, (X, C) has no regular completion; in other words, (X, C) $\& \mathrm{RCHY}$. Note that $R(X, C) \neq R^{\wedge}(X, C)$. Thus, under the assumptions of Section 3 , $M$ and $M^{\wedge}$ are in general distinct modification functors.

5. MORE COMPLETION FUNCTORS.

Let $(P)$ be a convergence space property which is both herditary (preserved under convergence subspaces) and productive (preserved by Cartesian products). Let $\underbrace{\mathrm{C} H}$ be the full category of $\mathrm{CHY}$ consisting of Cauchy subspaces of convergence spaces (considered as complete Cauchy spaces) which have property (P).

LEMMA 5.1. Let $(\mathrm{X}, \mathrm{C})$ be a Cauchy space such that there exists $\left(\mathrm{X}, \mathrm{C}^{\prime}\right) \in \underline{\mathrm{PCH}}$ with $\left(\mathrm{X}, \mathrm{C}^{\prime}\right) \leq(\mathrm{X}, \mathrm{C})$. Then there is a finest object

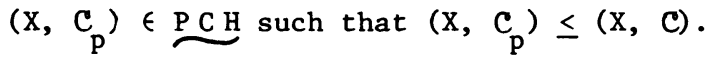

PROOF. Let $\left\{\left(\mathrm{X}, \mathrm{C}_{\alpha}\right): \alpha \in \mathrm{I}\right\}$ be the set of all objects in $\mathrm{PCH}$ coarser than $(X, C)$. Then each $\left(X, C_{\alpha}\right)$ is a Cauchy subspace of a convergence space $\left(Y_{\alpha}, q_{\alpha}\right)$ which has property $(P)$. Then $X$ can be regarded in a natural way as a subset of the Cartesian product $(\mathrm{Y}, \mathrm{q})$ of the family $\left\{\left(\mathrm{Y}_{\alpha}, \mathrm{q}_{\alpha}\right): \alpha \in I\right\}$. The Cauchy subspace $\left(X, C^{\prime \prime}\right)$ of $(Y, q)$ determined by $X$ is, by our assumption, a member of $\mathrm{PCH}$. One can easily verify that $\left(\mathrm{X}, \mathrm{C}^{\prime \prime}\right)=\left(\mathrm{X}, \mathrm{C}_{\mathrm{p}}\right)$ is the finest object in $\mathrm{PCH}$ coarser than $(\mathrm{X}, \mathrm{C})$.

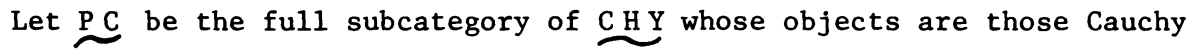

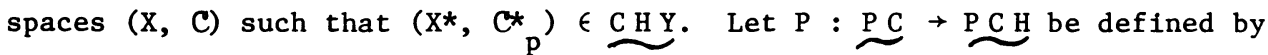
$P(X, C)=\left(X, C_{P}\right)$, and $P(f)=f$ for all morphisms $f \in \underline{P C}$. In order for $P$ to be a functor, it must have the following property: If $\mathrm{f}:(\mathrm{X}, \mathrm{C}) \rightarrow(\mathrm{Y}, \theta)$ is a morphism in $\underbrace{\mathrm{C}}$, then $\mathrm{f}: \mathrm{P}(\mathrm{X}, \mathrm{C}) \rightarrow \mathrm{P}(\mathrm{Y}, \mathbb{Q})$ is a morphism in $\underbrace{\mathrm{P} H}$.

THEOREM 5.2. If $P$ is a functor on $\underbrace{\mathrm{C}}$, then $\mathrm{P}$ is a modification functor which satisfies conditions $(\mathrm{L}),(\mathrm{H})$, and $(\mathrm{C})$. In this case, $\underbrace{\mathrm{PCH}}=\underbrace{\mathrm{PH}}_{\mathrm{CH}}$ the completion subcategory corresponding to the modification functor P, 
and $\mathrm{PW}$ is the completion functor on $\mathrm{PCH}$.

PROOF. If $P$ is a functor, then it is obviously a modification functor which satisfies condition (L). Furthermore, the arguments used to verify conditions (H) and (C) in the proof of Proposition 4.1 can be applied to show that $\mathrm{P}$ also satisfies these conditions. Note that $\mathrm{PCH}$ consists precisely of the P-spaces. Since each P-space is, by definition, a Cauchy subspace of complete P-space, it follows by Theorem 3.2 and Theorem 3.3 that PCY $=$ PCHY is the completion subcategory of $\mathrm{CHY}$ determined by $\mathrm{P}$, and $\mathrm{PW}$ the associated completion functor.

If $\mathrm{P}$ is a functor, then $\mathrm{P}=\mathrm{P}^{\wedge}$ (in the notation of Section 3 ), since every $\mathrm{P}$-space is in $\mathrm{PCHY}$. If $(\mathrm{P})$ is the property of being a regular convergence space, then $P$ is the modification functor $R^{\wedge}$ rather than $R$, but $\underbrace{P C H}=R C H Y$ and $\mathrm{PW}=\mathrm{RW}$ is the regular completion functor.

For the remainder of this section, we discuss the results of an earlier paper [3], in the light of the methods developed in Section 3 and 5 of this paper. The completion functors $\mathrm{N}$ and $\mathrm{N}_{\mathrm{S}}$ of [3] are both describable as $\mathrm{P} \mathrm{W}$, where $(P)$ is in the first case the C-embedded property of Binz (see [2]), and in the second case the sequential regularity of Novak (see [3] and [6]). Both of these properties are known to be hereditary and productive, and, in each case, $P$ is a modification functor. In the case where $(P)$ is the C-embedded property, the $\mathrm{P}$-spaces are the $\hat{\mathrm{C}}$-embedded spaces which were originally introduced and internally characterized in [4]. In the second case, the P-spaces are the sequentially regular Cauchy spaces which are defined and characterized in [3] .

The completely regular Cauchy spaces of [3] correspond to the category $\mathrm{PCH}$ in the case where $(\mathrm{P})$ is the property of being a completely regular topological space; by the results of this section they constitute a completion subcategory of $\mathrm{CHY}$. The category $\mathrm{UCH}$ of uniformizable Cauchy spaces described in [3] is also a completion subcategory of $\mathrm{CHY}$, but is not the form discussed 
in this section, since the convergence space property of "being compatible with a complete uniformity" is not hereditary. However if $U$ is the modification functor which assigns to each eligible Cauchy space the finest uniformizable Cauchy space coarser than itself, then U satisfies conditions of Section 3, and $\underline{\mathrm{UCH}}=\underline{\mathrm{UCHY}}$ is the associated completion subcategory of $\mathrm{CHY}$.

The completion functors determined by the completely regular and uniformizable Cauchy structures, begin toplogical, are clearly strict. It was shown in [3] that the completion functor $\mathrm{N}_{\mathrm{S}}$ associated with the sequentially regular Cauchy spaces is not strict. It would be desirable to find some general criterion for determining which of the completion functors generated by the methods described in this paper are strict. Also, in view of the examples of Section 4, it would be desirable to find an internal characterization for the Cauchy spaces which are members of RCHY.

\section{REFERENCES}

1. Berri, M. P., and R. H. Sorgenfrey, "Minimal Regular Spaces", Proc. Amer. Math. Soc. 14(1963), 454-458.

2. Binz, E., Continuous Convergence in $C(X)$, Lecture Notes in Mathematics 469, Springer-Verlag, Berlin-Heidelberg-New York 1975.

3. Frič, R., and D. C. Kent, "On the Natural Completion Functor for Cauchy Spaces", Bull. Astral. Math. Soc. 18(1978), 335-343.

4. Gazik, R. J., and D. C. Kent, "Regular Completions of Cauchy Spaces via Function Algebra", Bull. Astral. Math. Soc. 11(1974), 77-88.

5. Gazik, R. J., and D. C. Kent, "Coarse Uniform Convergence Spaces", Pacific J. Math. 61 (1975), 143-150.

6. Novak, "On Convergence Spaces and Their Sequential Envelopes", Czech. Math. J. 15(1965), 74-100.

7. Redfield, R. H., "Completing Convergence Structures", (Abstract 78T-G79), Notices Amer. Math. Soc. 25 (1978), A-488. 
8. Reed, E. E., "Completions of Uniform Convergence Spaces", Math. Ann. $\underline{194}(1971), 83-108$.

9. Richardson, G. D., and D. C. Kent, "Regular Compactifications of Convergence Spaces", Proc. Amer. Math. Soc. 31(1972), 571-573.

10. Wyler, 0., "Ein Komplettierunpfunktor für uniform Limesräume", Math Nachr. 46 (1970), 1-12. 


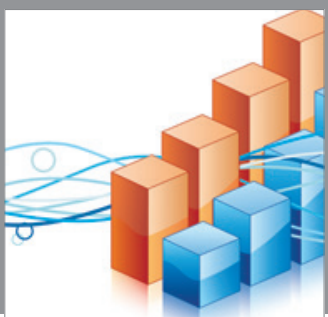

Advances in

Operations Research

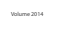

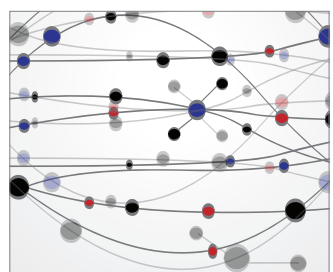

\section{The Scientific} World Journal
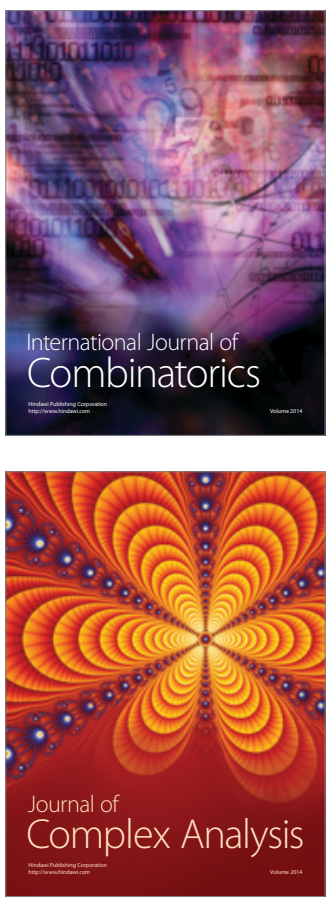

International Journal of

Mathematics and

Mathematical

Sciences
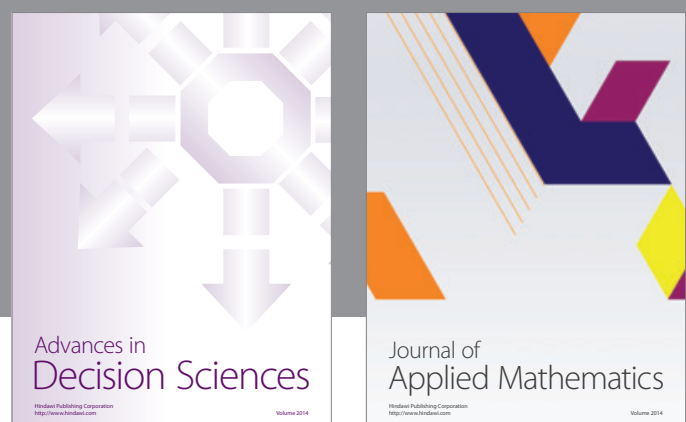

Journal of

Applied Mathematics
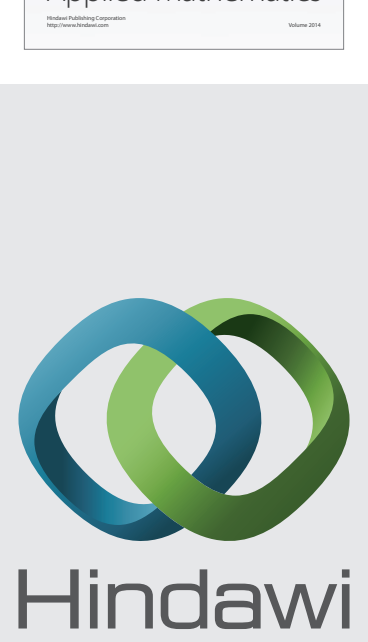

Submit your manuscripts at http://www.hindawi.com
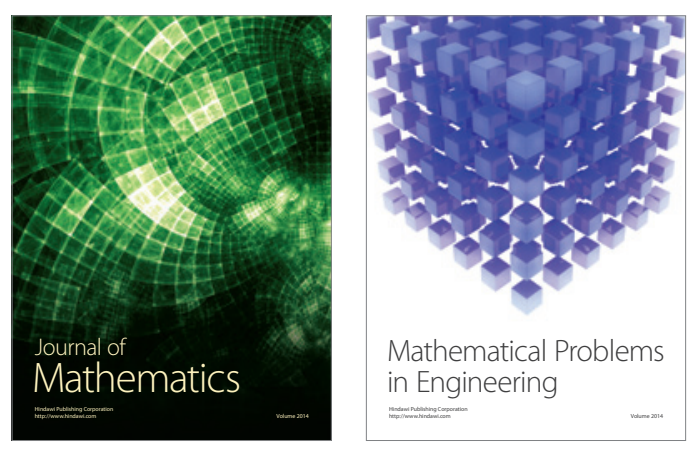

Mathematical Problems in Engineering
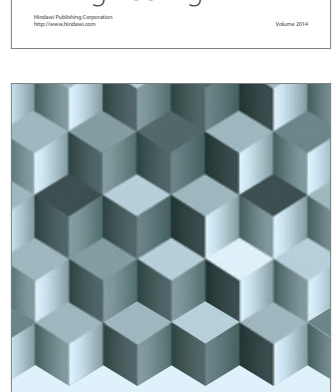

Journal of

Function Spaces
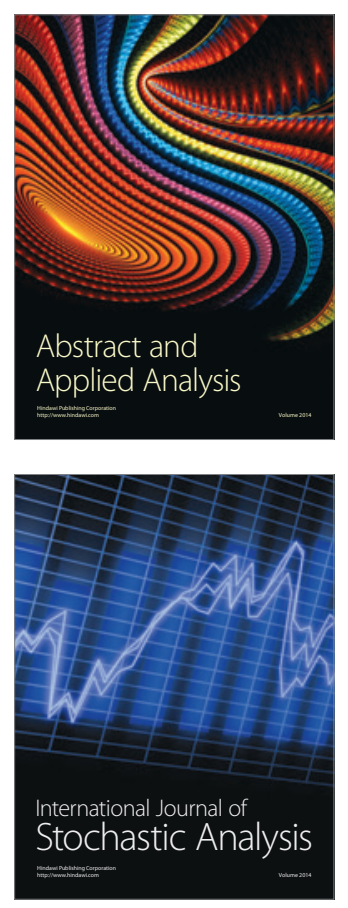

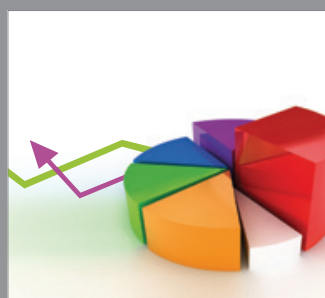

ournal of

Probability and Statistics

Promensencen
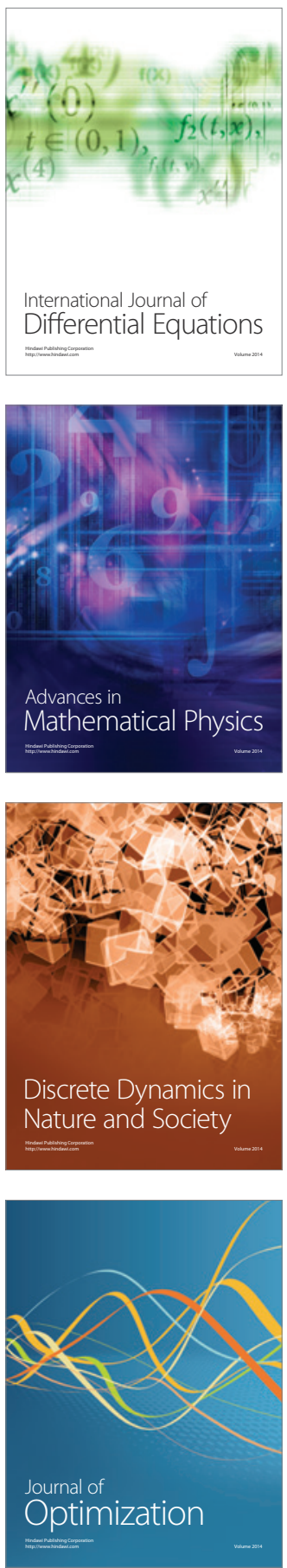